\title{
Quasiregular mapping that preserves plane
}

\author{
Anila Duka, Ndriçim Sadikaj \\ University “Ismail Qemali", \\ Vlora, Albania \\ dukaanila@gmail.com,ndsadikaj@gmail.com
}

ABSTRACT

Quasiregular mappings are a natural generalization of analytic functions to higher dimensions. Quasiregular mappings have many properties. Our work in this paper is to prove the following theorem: If $f=a+\bar{b}$ is a quasiregular mapping which maps the plane onto the plane, then $f$ is a bijection.

We do this by finding the connection between quasiregular and quasiconformal mappings.

\section{Keywords}

Quasiconformal mapping; quasiregular mapping.

\section{Academic Discipline}

Complex Analysis, Harmonic Analysis

\section{SUBJECT CLASSIFICATION}

Complex Analysis

\section{INTRODUCTION}

Quasiregular mappings are a natural generalization of analytic functions to higher dimensions. They are defined by condition on regularity and distortion. We begin with conformal mappings and their rigidity. We explain the need of new mappings such that quasiconformal mappings. In the other section we define quasiregular mappings and in the last section we prove the main theorem using the relation between quasiregular and quasiconformal mappings.

A quasiregular homeomorphism is called quasiconformal.

\section{PREMINALIRY}

Here we give some definitions and properties of conformal and quasiconformal mappings.

\subsection{CONFORMAL MAPPINGS}

Conformal mappings are very known in complex analysis. In every complex analysis book we can find them.

We can see [3] for conformal mappings.

Conformal mappings are the mappings that preserves the angle. Quasiconformal mappings are natural generalisation of conformal mappings. Let see the definition of conformal function.

\section{Definition 2.1}

Let $\Omega_{1}, \Omega_{2}$ be open subspaces of the plane $\square$.

The function $f: \Omega_{1} \rightarrow \Omega_{2}$ is called conformal if $f$ is bijective and analytic .

We say that, $f$ has complex derivative in $z_{0} \in \square$ where $f\left(z_{0}\right) \in \square$ we can write

$f(z)=f\left(z_{0}\right)+f^{\prime}\left(z_{0}\right)\left(z-z_{0}\right)+o\left(z-z_{0}\right)$. So, if $f$ is conformal in $z_{0}$ then $f$ has derivative in $\square$ with $\bar{\delta} f\left(z_{0}\right)=0, \delta f\left(z_{0}\right)=f^{\prime}\left(z_{0}\right) \neq 0$ and $\mu_{f}\left(z_{0}\right)=0$.

We say that the conformal map $f: \Omega_{1} \rightarrow \Omega_{2}$ maps infinitesimal small circles centered in $z_{0}$ to infinitesimal small circles centered in $f\left(z_{0}\right)$, although $f$ will be good approximated near $z_{0}$ related with

$L(z)=f\left(z_{0}\right)+f^{\prime}\left(z_{0}\right)\left(z-z_{0}\right)$, which maps such circles to such circles. 
Let see three properties of conformal deformation

1. Conformality implies smoothness.

2. Conformal transformations are holomorphic difeomorphisms and conversely for $n=2$.

3. Riemann Mapping Theorem.

But conformal mappings are also rigid and we can list the rigidity of they.

1. Liouville's theorem: For $n \geq 2$ conformal deformations are composition of translations, rotations, dilitations and inversions.

2. Rigidity on boundary value: In plane even we have Riemann Mapping Theorem, we can not describe the data boundary when we map conformaly a domain to another.

\subsection{QUASICONFORMAL MAPPINGS}

For quasiconformal mappings we can see [1], [2], [4], [7], [8], etc.

A sense preserving homeomorphism $g: \Omega \rightarrow \Omega_{0}$ where $\Omega$ and $\Omega_{0}$ are subdomains of the complex plane is said to be k-quasiconformal if $g$ is absolutely continuous on almost all horizontal and almost all vertical lines in $\Omega$ and if there exists a constant

$k \in[0,1)$ such that

$\left|g_{\bar{z}}(z)\right| \leq k\left|g_{z}(z)\right|$, for almost all $z \in \Omega$.

The last requirement is equivalent to

$\left|g_{x}(z)\right|^{2}+\left|g_{y}(z)\right|^{2} \leq\left(K+\frac{1}{K}\right) J_{f}(z)$, for almost all $z \in \Omega$, where $J_{g}$ is the Jacobian of $g$ and $K=\frac{1+k}{1-k} \geq 1$.

Quasiconformality is a degree of ordness: the right one for studying the stability of the structure of holomorphic mappings. Topological conjugations between holomorphic mappings can be improved with quasiconformal mappings.

1. Quasiconformality implies $\square^{0}$

2. Quasiconformality does not implies $\square^{1}$ and $\square^{1}$ does not implies quasiconformality.

3. They are very flexible, hence, good to construct model.

\section{QUASIREGULAR MAPPING}

In [6] we see quasiregular mapping and some properties. We can refer also [9],[10].

A continuous function $f: G \rightarrow \square^{n}, n \geq 2$ from a domain

$G$ in $\square^{n}$ is called quasiregular, if $f$ is in the Sobolev space.

$W_{l o c}^{1, n}(G)$ and there is a constant $K, 1 \leq K \leq \infty$ such that the inequality holds in $G$

$\left|f^{\prime}(x)\right|^{n} \leq K J_{f}(x)$

Where, $f^{\prime}(x)$ is the formal derivative of $f$ and $\left|f^{\prime}(x)\right|=\max _{|h|=1}\left|f^{\prime}(x) h\right|$.

The smallest $K \geq 1$, that this inequality is true is called the outer dilatation of $f$ and it is signed by $K_{O}(f)$.

If $f$ is quasiregular, then the smallest $K \geq 1$, that this inequality: 
$J_{f}(x) \leq K l\left(f^{\prime}(x)^{n}\right)$

Is true almost everywhere in $G$, is called the inner dilatation of $f$ and it is signed by

$K_{I}(f)$, where $l\left(f^{\prime}(x)\right)=\min _{|h|=1}\left|f^{\prime}(x) h\right|$.

The maximal dilatation of $f$ is the number $K(f)=\max \left\{K_{I}(f), K_{O}(f)\right\}$.

If $K \leq K(f), f$ is called K-quasiregular.

A homeomorphism quasiregular $f: G \rightarrow f G$ is called quasiconformal.

From generalization of Liouville's Theorem, for $n \geq 3$ every mapping 1-quasiregular in

$\square^{n}$ is a of Mobius transformation or is a constant.

Mobius transformations are very useful in studying quasiregular mapping.

\section{Definition 4.1:}

A quasiregular map is the composition $f^{\circ} \varphi$, where $\varphi$ is

Quasiconformal in the plane and $f$

is rational.

\section{Lemma 4.1:}

Let $g$ be a quasiregular mapping. If $g: U \rightarrow V$ is surjective and

$v$ is a Beltrami coeficent in $V$ then there is an only Beltrami coeficent

$\eta$ in $U$ such that: $g: U[\eta] \rightarrow V[v]$ is analytic. We denote

$\eta=g^{*} v$ and we call $\eta$ pulling back $v$ by $g$.

\section{Lemma 4.2:}

Assume that surjective mapping $g: \bar{\square} \rightarrow \bar{\square}$ is continuous and locally quasiconformal except a finite set of points

$K$ ( not necessary global homeomorphism ). If $\left\|\mu_{g}\right\|_{\infty}<1$, then $g$ is quasiregular.

Note:

This lemma shows us that quasiregular mappings are the mappings that are globally continuous, but quasiconformal locally except a finite set of points .

(Likewise rational mappings that are globally continuous, but conformal locally except a finite set of points .)

\section{THE MAIN THEOREM}

\section{Theorem 5.1:}

If $f=a+\bar{b}$ is a quasiregular mapping which maps the plane onto the plane, then $f$ is a bijection.

\section{Proof.}

We can refer [5] because we have the analogue theorem there.

Quasiregular mappings are a natural generalization of analytic functions to higher dimensions.

They are defined by condition on regularity and distortion. A quasiregular homeomorphism is called quasiconformal. 
We can say that $f$ is quasiregular if $\left|b^{\prime}\right|<k\left|a^{\prime}\right|$. Here we want to show the conection between quasiregular and quasiconformal mappings. We find that this function has a discrete and finite set of points, where the function is not injective, so we show that this is 1 quasiconformal, so it is conformal, so it is analytic and bijective. Reshetnyak gave us a definition for mappings of bounded distortion or, as they are more commonly called today, quasiregular mappings.

\section{Definition 5.1:}

We call a continuous map $f: \Omega \rightarrow X$ quasiregular if $f$ is constant, or

1. $f$ is sense preserving, discrete and open

2. there exists $H<\infty$, so that $H_{f}(x) \leq H$ for almost every $x \in \Omega$

3. and, $H_{f}(x) \leq \infty$, for every $x \in \Omega$

We call a f: $\Omega \rightarrow X$ monotone if the following holds true with $T=1$ $\operatorname{diam} f(B(x, r)) \leq T \operatorname{diam} f(S(x, r))$

for every $\mathrm{B}(\mathrm{x}, \mathrm{r}) \subset \subset \Omega$

If there exists $1 \leq T<\infty$ such that the inequality is satisfied, then the mapping $f$ is called pseudomonotone. We can see also the Stoilow factorization.

Theorem 5.2: (Stoilow factorization)

Let $\mathrm{f}: \mathrm{C} \rightarrow \mathrm{C}$ be a quasiregular mapping. Then there exists an analytic function $\mathrm{g}$ and a quasiconformal mapping $\mathrm{h}$ such that $f=g \circ h$.

\section{Corollary 5.1:}

Let $\mathrm{f}: \mathrm{C} \rightarrow \mathrm{C}$ be quasiregular. Then $\mathrm{B}(\mathrm{f})$ the set of branch points, is a discrete set of points. In particulary if $f$ is quasiregular of polynomial type, then $B(f)$ is a finite set of points.

The theorem 3.3 of Kovalev gives a sharp bound for $|f(z)|$.

\section{Theorem 5.3 :}

Let $\mathrm{f}: \mathrm{D} \rightarrow \mathrm{D}$ be a K-quasiregular mapping such that,

$\mathrm{f}(0)=0$. Then for every $z$ ò $D$

$$
|f(z)| \leq\left(1+\omega_{f}(0) \omega_{f}(z)|z|^{-2 / k}\left(1-|z|^{2}\right)^{1 / k}\right)^{-1 / 2} \text {. }
$$

For every $K \geq 1$ and for every $z \in D$ there exists a $\mathrm{K}$-quasiconformal mapping for which equality is attained.

Where $\omega_{f}\left(z_{0}, \delta\right)$ for $z_{0} \in D$, and small $\delta>0$ the local modulus of continuity is majorized by $A \delta^{1 / k}$ for some positive $A$

$\omega_{f}\left(z_{0}, \delta\right)=\max \left\{\left|f(z)-f\left(z_{0}\right)\right|: z \in D,\left|z-z_{0}\right| \leq \delta\right\}$. So locally this function is homeomorphism.

1-quasiconformal mapping is conformal mapping, which means that it is analytic and bijective.

So what we wanted to prove.

\section{REFERENCES}

1. [L. V. AHLFORS, Lectures on quasiconformal mappings, University Lecture Series 38(2006), Amer. Math. Soc., Providence, RI].

2. Harmonic and Quasiconformal Mappings. David Kalaj and Miodrag Mateljevi'c (HQM2010),Proceedings of the ICM2010 Satellite Conference, J. Analysis, Volume 18 (2010), 239-260.

3. [Juha Heinonen What is a conformal mapping?, Notices of the AMS, volume 53, Nr 11].

4. Description of harmonic quasiconformal mappings. A.Duka, http://eujournal.org/index.php/esj/article/view/2444, 2013.

5. On quasiconformal selfmappings of the unit disk and the unit ball, A. Duka, N. Sadikaj. CMM 2016 Macedonia.

6. Mapping problems for quasiregular mapping. M. Huang, A. Rasila, X. Wang. arxiv.org 29 Nov 2012. 
7. Quasiconformal harmonic mapping between Jordan domains\}, D. Kalaj, Math. Z. 260(2)(2008), 237-252.

8. Inner estimate and quasiconformal harmonic maps between smooth domains\}, D. Kalaj and M. Mateljevic, Journal d'Analise Math. 100(2006), 117-132.

9. Conformal geometry and quasiregular mappings, (Lecture Notes in Mathematics), M. Vourinen, $1988^{\text {th }}$ Edt.

10. Infinitesimal geometry for quasiregular mappings, V. Ya. Gutlyanksii, O. Martio, V. I. Ryazanov, M. Vourinen, Ann, Acad, Scien, Fennic. Vol 25,2000. 